\title{
Direct Observation of Symmetry-Dependent Electron-Phonon Coupling in Black Phosphorus
}

Nannan Mao, ${ }^{\dagger, \star}, \|$ Xingzhi Wang, ${ }^{\ddagger},{ }^{\prime}, \|$ Yuxuan Lin, ${ }^{\dagger}$ Bobby G. Sumpter,,${ }^{\perp,}$ Qingqing Ji, ${ }^{\dagger}$ Tomás Palacios, ${ }^{\dagger}$ Shengxi Huang, ${ }^{\circ}$ Vincent Meunier, ${ }^{\nabla}$ Mildred S. Dresselhaus, ${ }^{\dagger}$ William A. Tisdale, ${ }^{\diamond}$ Liangbo Liang, ${ }^{*}, \perp$ Xi Ling, $,{ }^{*}, \S, \otimes$ and Jing Kong ${ }^{*}, \dagger$

${ }^{\dagger}$ Department of Electrical Engineering and Computer Science and ${ }^{\diamond}$ Department of Chemical Engineering, Massachusetts Institute of Technology, Cambridge, Massachusetts 02139, United States

${ }^{\dagger}$ Department of Chemistry and ${ }^{\S}$ Division of Materials Science and Engineering, ${ }^{\otimes}$ The Photonics Center, Boston University, Boston, Massachusetts 02215, United States ${ }^{\perp}$ Center for Nanophase Materials Sciences and ${ }^{\#}$ Computational Sciences and Engineering Division, Oak Ridge National Laboratory, Oak Ridge, Tennessee 37831, United States

${ }^{\circ}$ Department of Electrical Engineering, The Pennsylvania State University, University Park, Pennsylvania 16802, United States

DDepartment of Physics, Applied Physics, and Astronomy, Rensselaer Polytechnic Institute, Troy, New York 12180, United States

"These authors contributed equally to this work.

*Corresponding authors: jingkong@mit.edu; xiling@bu.edu; liangl1@,ornl.gov; 


\section{This PDF file includes:}

Section S1: Identifying the crystalline orientations of black phosphorus (BP) using angle-dependent Raman frequency.

Section S2: Polarization and excitation-energy-dependent Raman spectra of BP. Section S3: The contribution of the optical interference enhancement to the resonance Raman excitation profiles (REP) of the $A_{g}$ modes in BP.

Section S4: Raman intensity ratio $\left(\mathrm{I}_{\mathrm{ZZ}} / \mathrm{I}_{\mathrm{AC}}\right)$ of the $\mathrm{A}_{\mathrm{g}}$ modes as a function of the excitation energies for BP samples with the different thicknesses.

Section S5: Thickness-dependent intensity polar plots of the $\mathrm{Ag}_{\mathrm{g}}{ }^{1}$ mode under the excitation energy between $2.60-2.73 \mathrm{eV}$.

Section S6: Density functional theory (DFT) calculations of the resonance Raman excitation profiles

Figure S1. Angle-dependent Raman frequency of BP.

Figure S2. Polarization and excitation-energy-dependent Raman spectra of BP. Figure S3. Interference enhancement for $7.7 \mathrm{~nm} \mathrm{BP}$.

Figure S4. Excitation-energy-dependent interference enhancement factors for BP with different thicknesses.

Figure S5. Intrinsic Raman excitation profiles of BP with different thicknesses. Figure S6. Thickness-dependent Raman anisotropy of BP.

Table S1. Thickness-dependent Raman intensity polar plots of the $\mathrm{A}_{\mathrm{g}}{ }^{1}$ mode under the excitation energy at 2.60, 2.66 and $2.73 \mathrm{eV}$. 


\section{Section S1: Identifying the crystalline orientations of BP using angle-dependent Raman frequency.}

(a)
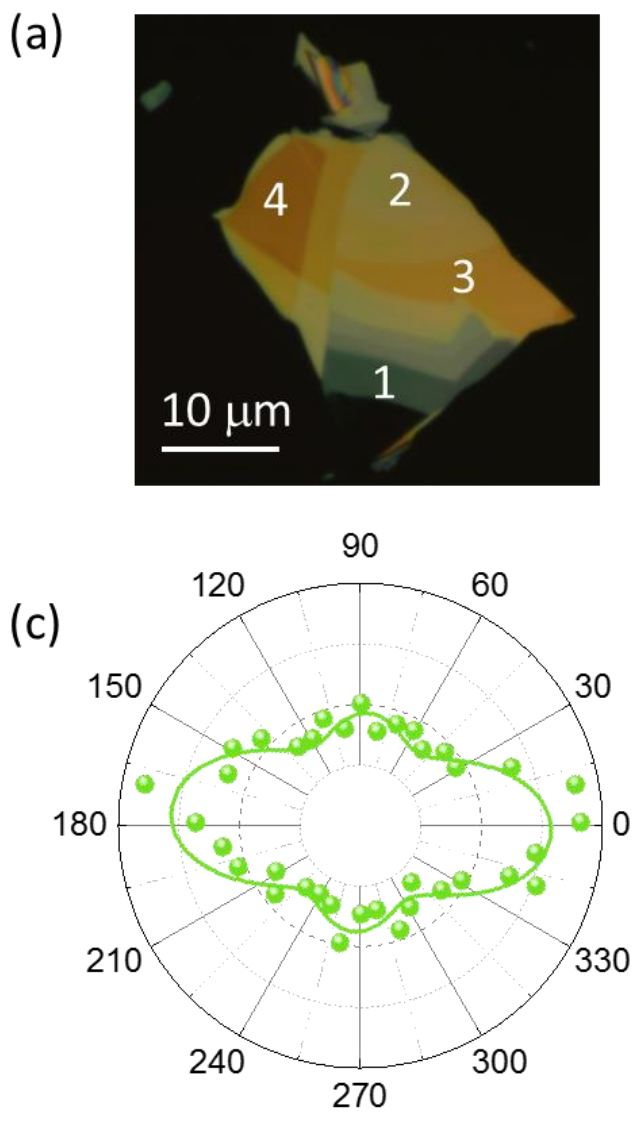

(b)

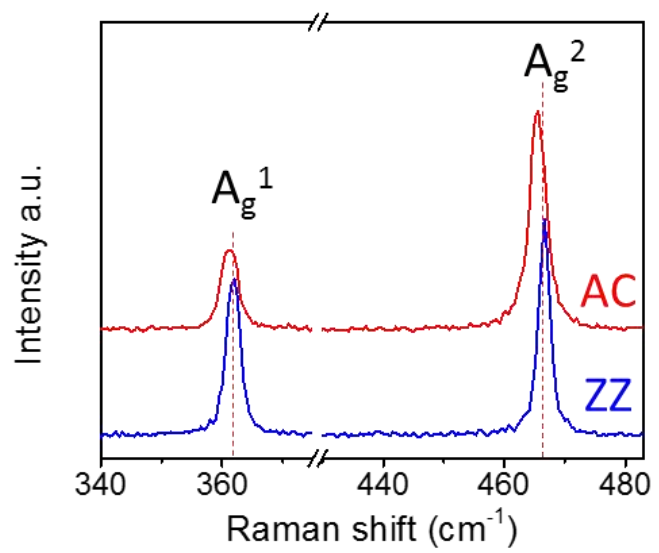

(d)

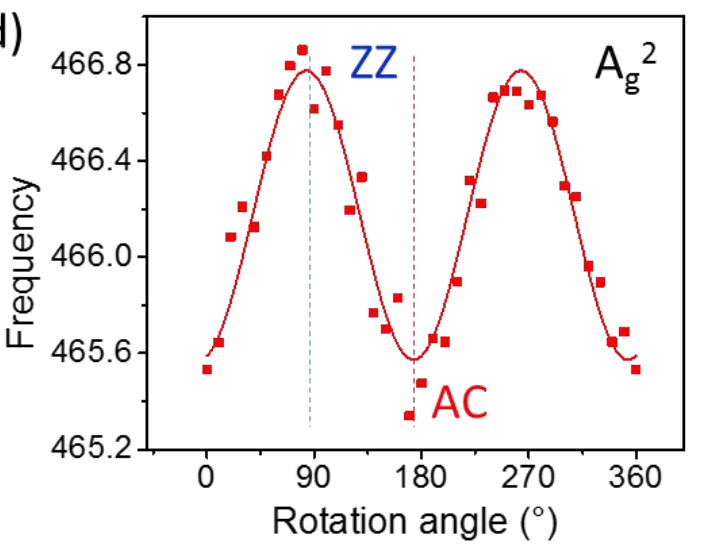

Figure S1. Angle-dependent Raman frequency of BP. (a) Optical image of a BP flake on a fused silica substrate, with four regions marked 1-4. (b) Polarized Raman spectra of BP in region 4 (R4) with P//AC (red) and P//ZZ (blue), under the excitation energy at 2.18 $\mathrm{eV}$. The dashed lines are guides for the eyes. (c and d) Angle-dependent Raman intensities (c) and frequencies (d) of the $\mathrm{Ag}_{\mathrm{g}}{ }^{2}$ mode for the same BP under parallel polarization. Here $0^{\circ}\left(90^{\circ}\right)$ corresponds to the $\mathrm{AC}(\mathrm{ZZ})$ direction of $\mathrm{BP}$.

Figure S1a is the optical image of a BP sample exfoliated on a fused quartz substrate. Four regions which belong to the same crystalline orientation are labeled as $1-4$. The thickness increases from region $1(\mathrm{R} 1,10 \mathrm{~nm})$ to region $4(\mathrm{R} 4,50 \mathrm{~nm})$. In order to 
identify the crystalline orientations of this BP flake, angle-dependent polarized Raman spectra were measured on a triple grating Raman system under the parallel polarization. The intensity polar plot of the $\mathrm{Ag}_{\mathrm{g}}{ }^{2}$ mode of $\mathrm{R} 4$ under the excitation energy at $2.18 \mathrm{eV}$ shows a main axis along $0^{\circ}$ (Figure $\mathrm{S} 1 \mathrm{c}$ ), which corresponds to either the $\mathrm{AC}$ or $\mathrm{ZZ}$ direction of BP. ${ }^{1-3}$ However, we cannot determine precisely which direction (AC or ZZ) the sample is oriented along, due to the controversial reports of identifying BP's crystalline orientations using angle-dependent Raman intensity, as it depends on both the thickness and excitation energy. ${ }^{1,2,4,5}$ Raman spectra of BP in R4 with $\mathrm{P} / / 0^{\circ}$ (red) and $\mathrm{P} / / 90^{\circ}$ (blue) are presented in Figure S1b. It can be seen that the Raman spectra are dominated by two Raman peaks, $\mathrm{A}_{\mathrm{g}}{ }^{1}\left(363 \mathrm{~cm}^{-1}\right)$ and $\mathrm{A}_{\mathrm{g}}{ }^{2}\left(467 \mathrm{~cm}^{-1}\right)$. Both of them show obvious redshift at $0^{\circ} / 180^{\circ}$ (red) compared with that at $90^{\circ} / 270^{\circ}$ (blue), which is related to heating effect of the incident laser. ${ }^{6}$ Since the larger (smaller) absorption of BP for the incident laser and slower (faster) dissipation speed of the heat, the red (blue) spectrum corresponds to the $\mathrm{AC}(\mathrm{ZZ})$ direction of $\mathrm{BP} .^{7}$ The crystalline orientation of this sample was further confirmed by periodic variation patterns of the angle-dependent Raman frequencies in Figure S1d.

\section{Section S2: Excitation-energy-dependent polarized Raman spectra of BP with the laser polarizations along the armchair (P//AC) and zigzag (P//ZZ) directions.}



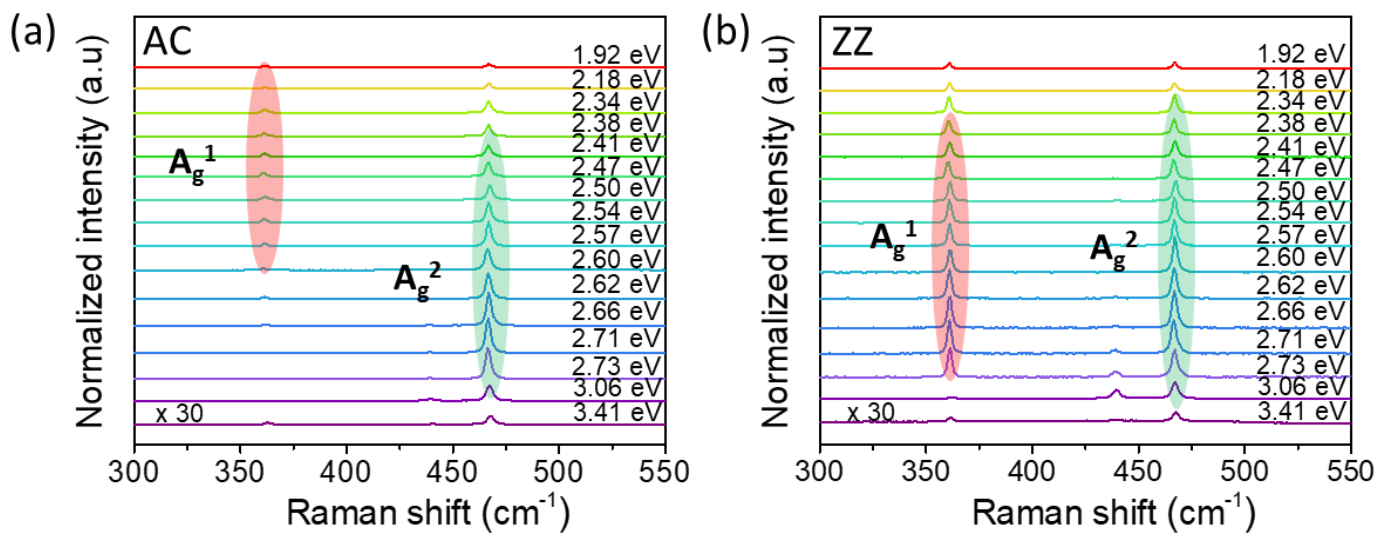

Figure S2. Polarization and excitation-energy-dependent Raman spectra of BP. (a and b) Excitation-energy-dependent polarized Raman spectra of a $7.7 \mathrm{~nm}$ BP with the laser polarizations along the $\mathrm{AC}$ (a) and $\mathrm{ZZ}$ (b) directions. The red and green ovals represent roughly the resonance regions for $\mathrm{Ag}_{\mathrm{g}}{ }^{1}$ and $\mathrm{Ag}_{\mathrm{g}}{ }^{2}$. The Raman intensities are normalized by the Raman peak of single-crystal quartz at $465 \mathrm{~cm}^{-1}$. Raman spectra under $3.41 \mathrm{eV}$ excitation are multiplied by 30 times for comparison.

The Raman intensities of both $\mathrm{Ag}_{\mathrm{g}}{ }^{1}$ and $\mathrm{Ag}_{\mathrm{g}}{ }^{2}$ at $3.41 \mathrm{eV}$ excitation are very weak. It is attributed to the weaker electron-phonon coupling in UV region than the visible region for both $\mathrm{A}_{\mathrm{g}}$ phonons. The absorption of BP in UV region is stronger than that of the visible region, ${ }^{8,9}$ suggesting that the electron-photon coupling might also be strong for UV excitation energy. In addition, the $\mathrm{B}_{2 \mathrm{~g}}$ mode is symmetry-forbidden under the parallel polarization when laser polarization is along the crystalline orientations of BP. Here the $\mathrm{B}_{2 \mathrm{~g}}$ mode is noticeable at $3.41 \mathrm{eV}$ excitation, which could be due to the stronger electron-phonon coupling under UV laser excitation and the slight deviation of the incident laser polarization with respect to the crystalline orientations of BP.

\section{Section S3: The contribution of the optical interference enhancement to the resonance Raman excitation profiles (REPs) of the Ag mode in BP.}


For BP samples on a fused silica substrate, due to multiple reflections in BP layer, there are multiple chances for BP layer to reabsorb the reflected incident light and scattered Raman light. A constructive or destructive interference effect will be caused due to the phase differences among the multiple reflected lights ${ }^{10}$. It will affect the practical Raman intensity of BP when the sample thickness is comparable with the wavelength of the incident laser. ${ }^{2,4}$ Since the refractive indices of BP are different along the AC and ZZ directions, the interference effect has distinct contributions to the Raman intensity of BP with the laser polarization along these two crystalline orientations. The multiple reflections induced net absorption $F_{e x}$ of excitation laser at depth $\mathrm{x}$ in the BP layer is presented by: ${ }^{10}$

$$
F_{e x}=t_{01} \frac{\left[1+r_{12} r_{23} e^{-2 i \beta_{2}^{e x}}\right] e^{-i \beta_{x}^{e x}}+\left[r_{12}+r_{23} e^{-2 i \beta_{2}^{e x}}\right] e^{-i\left(2 \beta_{1}^{e x}-\beta_{x}^{e x}\right)}}{1+r_{12} r_{23} e^{-2 i \beta_{2}^{e x}}+\left(r_{12}+r_{23} e^{-2 i \beta_{2}^{e x}}\right) r_{01} e^{-2 i \beta_{1}^{e x}}}
$$

where $t_{k l}=2 n_{k} /\left(n_{k}+n_{l}\right)$ and $r_{k l}=\left(n_{k}-n_{l}\right) /\left(n_{k}+n_{l}\right)$ are the transmission and reflection coefficients for the interfaces between $k$-th medium and $l$-th medium. Here we consider the system of BP on a fused silica substrate as four-media system: air (0), BP (1), $1 \mathrm{~mm}$ fused silica (2), and air (3). $n_{k}$ and $d_{k}$ are the complex refractive index and thickness for the $k$-th medium, respectively. The refractive indices of air $\left(n_{0}=n_{3}=1\right)$ and fused silica were taken from a previous report. ${ }^{11}$ Here the refractive indices of BP were taken from previous theoretical report. ${ }^{12} \beta_{x}^{e x}=2 \pi x n_{1} / \lambda_{e x}$ is phase difference of the excitation laser at depth $\mathrm{x}$ of BP layer, and $\beta_{k}^{e x}=2 \pi d_{k} n_{k} / \lambda_{e x}$ are the phase differences of the excitation laser in $k$-th medium. $\lambda_{e x}$ is the excitation wavelength.

Similarly, the net absorption for the scattered light $F_{s c}$ due to multiple reflection at depth $\mathrm{x}$ in the BP layer is expressed as: ${ }^{10}$

$$
F_{s c}(\mathrm{x})=t_{10} \frac{\left[1+r_{12} r_{23} e^{-2 i \beta_{2}^{s c}}\right] e^{-i \beta_{x}^{s c}}+\left[r_{12}+r_{23} e^{-2 i \beta_{2}^{s c}}\right] e^{-i\left(2 \beta_{1}^{s c}-\beta_{x}^{s c}\right)}}{1+r_{12} r_{23} e^{-2 i \beta_{2}^{s c}}+\left(r_{12}+r_{23} e^{-2 i \beta_{2}^{s c}}\right) r_{01} e^{-2 i \beta_{1}^{s c}}}
$$


Where $\beta_{x}^{s c}=2 \pi x n_{1} / \lambda_{s c}$ is the phase difference of the scattered laser at depth $\mathrm{x}$ of $\mathrm{BP}$ layer, $\beta_{k}^{s c}=2 \pi d_{k} n_{k} / \lambda_{s c}$ is phase differences of the scattered laser in $k$-th medium. $\lambda_{s c}$ is the wavelength of Raman scattering signal. For simplification, we consider the wavelengths of the Raman scattered light for the $\mathrm{Ag}_{\mathrm{g}}{ }^{1}$ and $\mathrm{Ag}_{\mathrm{g}}{ }^{2}$ phonons are the same. The total interference enhancement factor is given by: ${ }^{10}$

$$
F=\int_{0}^{d_{1}}\left|F_{e x}(x) F_{s c}(x)\right|^{2} d x
$$
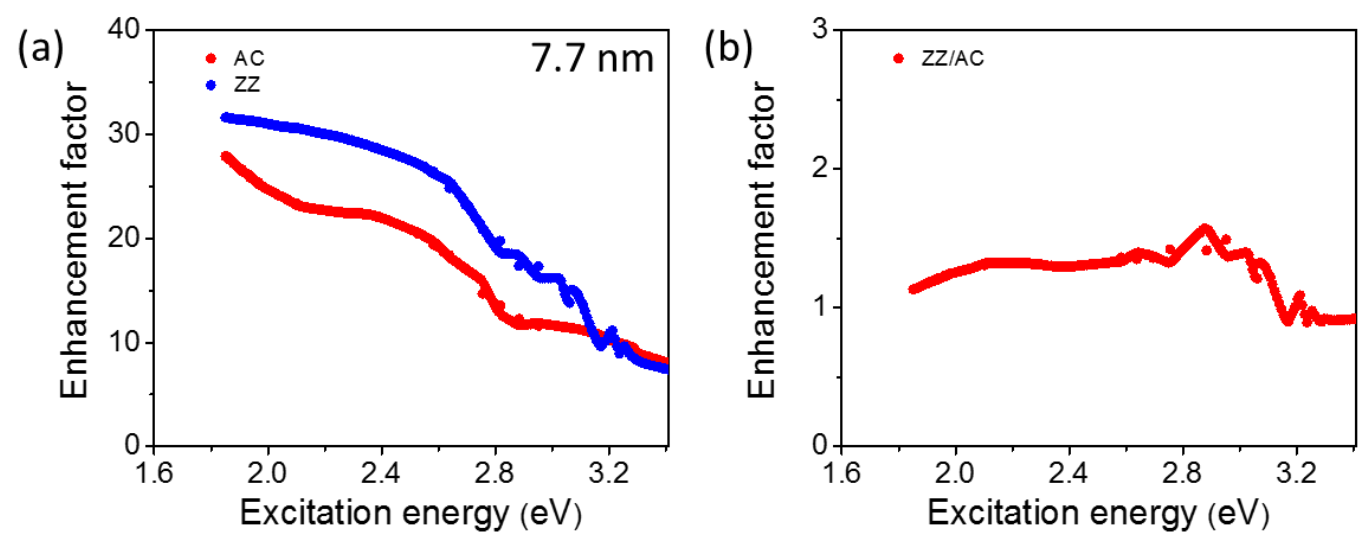

Figure S3. Interference enhancement for the $7.7 \mathrm{~nm}$ BP. (a) Excitation-energy-dependent interference enhancement factors for the $7.7 \mathrm{~nm} \mathrm{BP}$ on a fused silica substrate with $\mathrm{P} / / \mathrm{AC}$ (red line) and P//ZZ (blue line). (b) Interference enhancement ratio (ZZ/AC) between the $\mathrm{ZZ}$ and $\mathrm{AC}$ directions for the same sample. 

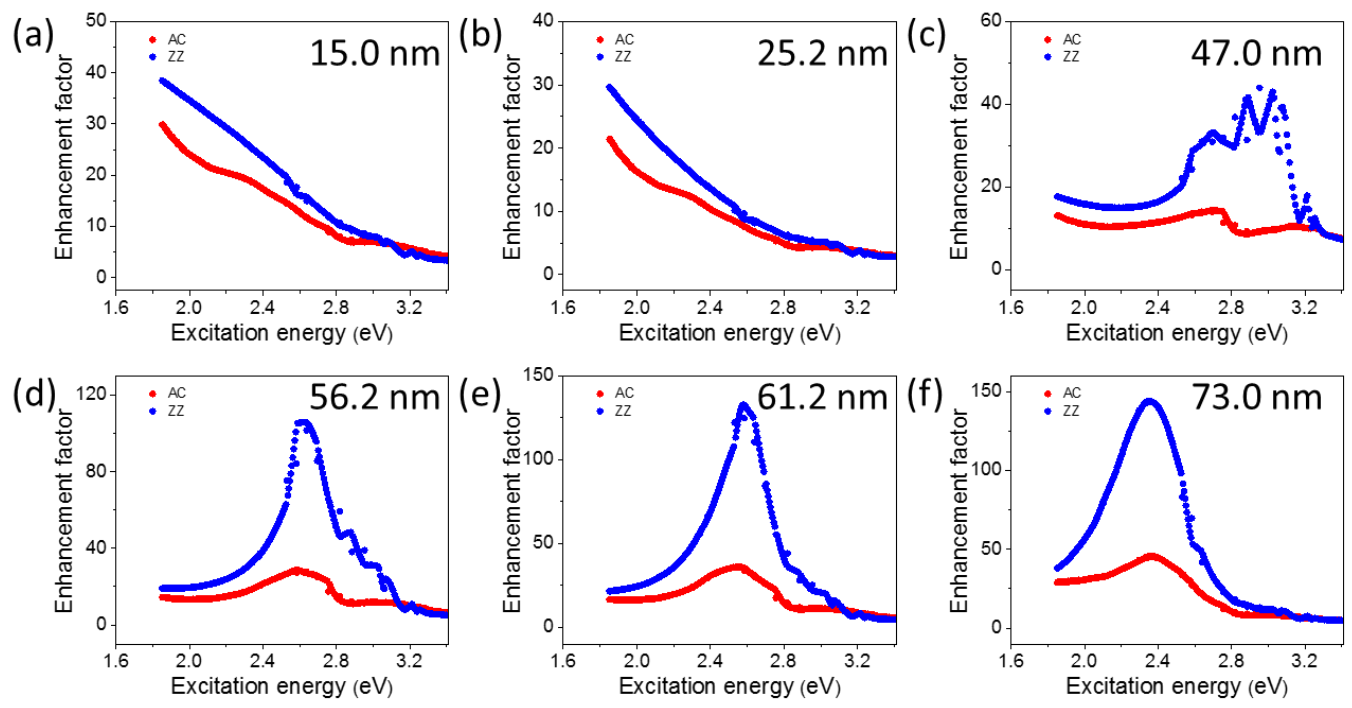

Figure S4. Simulated excitation-energy-dependent interference enhancement factors for BP with different thicknesses. (a, b, c, d, e and f) Simulated excitation-energy-dependent interference enhancement factors with P//AC (red line) and P//ZZ (blue line) for BP with different thicknesses on fused silica substrates: 15.0, 25.2, 47.0, 56.2, 61.2 and $73.0 \mathrm{~nm}$.
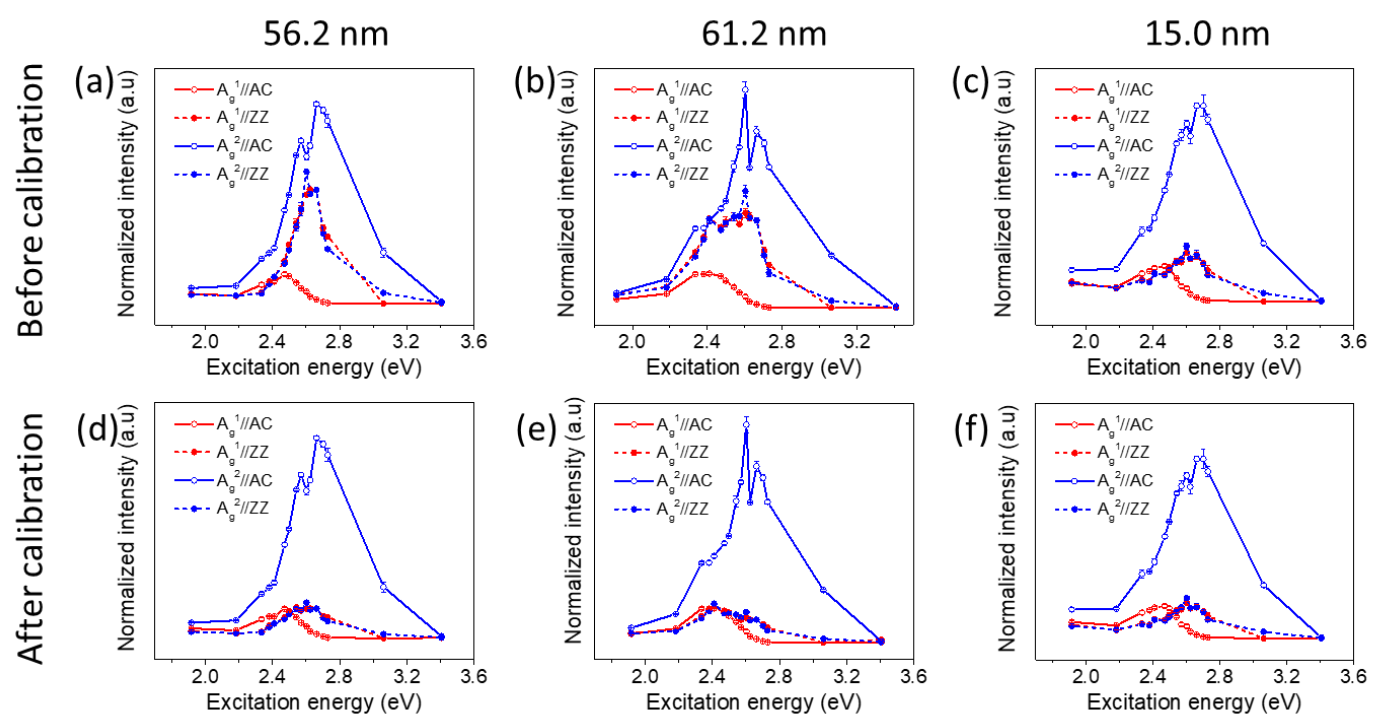

Figure S5. Intrinsic Raman excitation profiles of BP with different thicknesses. (a, b, c, $\mathrm{d}$, e and $\mathrm{f}$ ) REPs of the $\mathrm{A}_{\mathrm{g}}{ }^{1}$ (red lines) and $\mathrm{A}_{\mathrm{g}}{ }^{2}$ (blue lines) phonons in BP with P//AC (solid lines) and P//ZZ (dashed lines), before (a, b and c) and after calibration of 
interference effect (d, e and f). The thicknesses of BP are $56.2 \mathrm{~nm}$ (a and d), $61.2 \mathrm{~nm}(\mathrm{~b}$ and e) and $15.0 \mathrm{~nm}$ (c and f).

Section S4: Raman intensity ratio (Izz/I $/ \mathrm{AC}$ ) of the $A_{\text {g }}$ modes between P//ZZ and P//AC as a function of the excitation energies for BP samples with the different thicknesses.

(a)

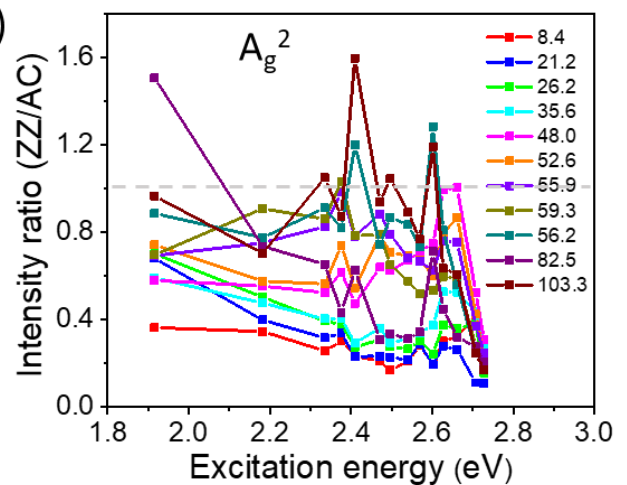

(b)

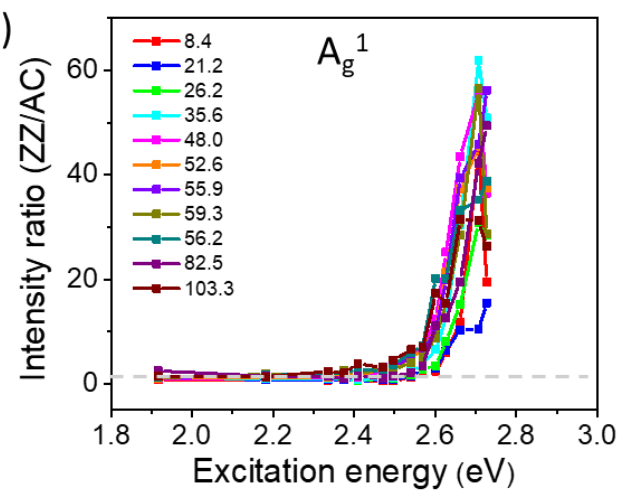

Figure S6. Thickness-dependent Raman anisotropy of BP. (a and b) Raman intensity ratios $\left(\mathrm{I}_{\mathrm{ZZ}} / \mathrm{I}_{\mathrm{AC}}\right)$ of the $\mathrm{Ag}_{\mathrm{g}}{ }^{2}$ (a) and $\mathrm{A}_{\mathrm{g}}{ }^{1}$ (b) modes with $\mathrm{P} / / \mathrm{ZZ}$ and $\mathrm{P} / / \mathrm{AC}$ as a function of the excitation energies for BP samples with the thickness of 8.4-103.3 nm. The solid lines are guides to the eye. The horizontal dashed lines in both panels indicate the intensity ratio $\mathrm{A}_{\mathrm{g}}\left(\mathrm{I}_{\mathrm{ZZ}} / \mathrm{I}_{\mathrm{AC}}\right)=1$.

Section S5: Thickness-dependent intensity polar plots of the $\mathrm{Ag}^{1}$ mode under the excitation energy between $2.60-2.73 \mathrm{eV}$.

Table S1. Thickness-dependent intensity polar plots of the $\mathrm{Ag}^{1}$ mode under the excitation energy at 2.60, 2.66 and $2.73 \mathrm{eV}$. The thicknesses of the BP samples range from $8.5 \mathrm{~nm}$ to $103.3 \mathrm{~nm}$. 


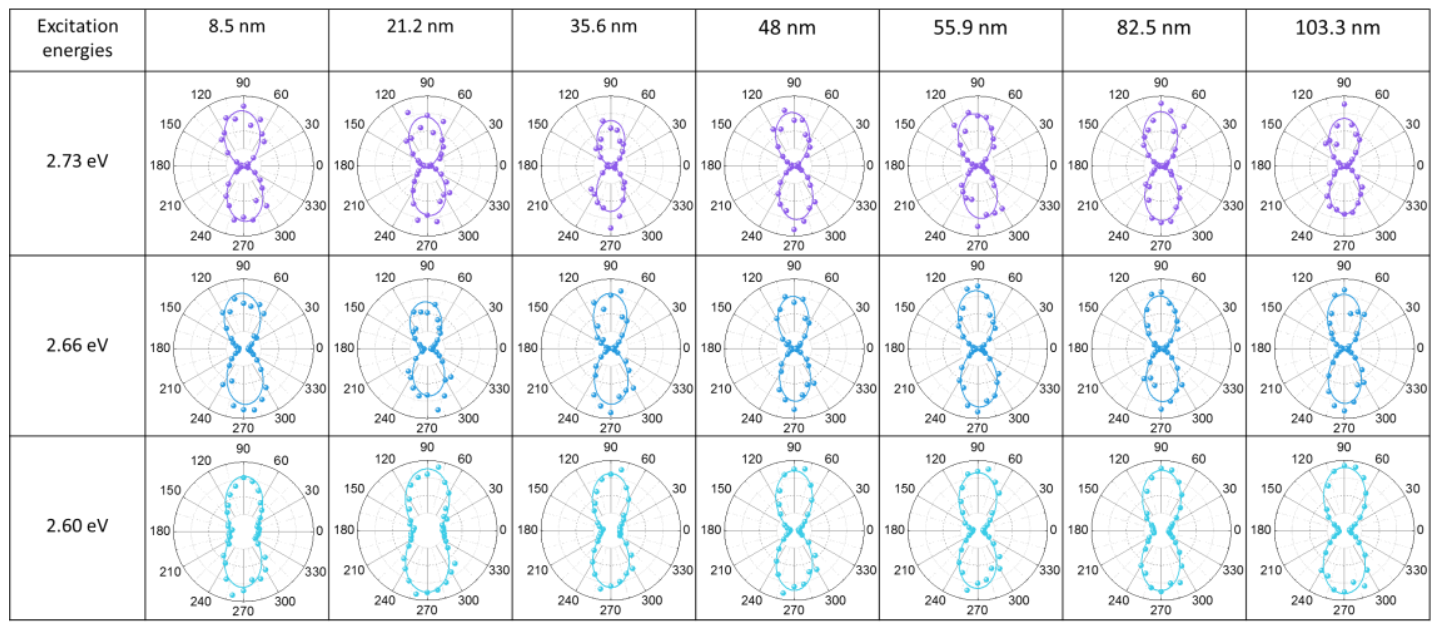

\section{Section S6: Density functional theory (DFT) calculations of the resonance Raman profiles}

First-principles DFT calculations were performed using the plane-wave VASP software. ${ }^{13}$ Projector augmented wave (PAW) pseudopotentials were used for electronion interactions and the Perdew-Burke-Ernzerhof (PBE) functional was selected for exchange-correlation interactions. ${ }^{14}$ Van der Waals (vdW) interactions between BP layers were described via the vdW density functional method optB86b-vdW. ${ }^{15}$ For bulk BP, where a cutoff energy was set at $500 \mathrm{eV}$ and a 9x12x4 k-point sampling was used, both atomic coordinates and lattice constants were optimized until the residual forces were below $0.001 \mathrm{eV} / \AA$. Raman calculations were then performed using the optimized bulk structure. The dynamical matrix was calculated via the finite difference method as implemented in the Phonopy software ${ }^{16}$ based on a $3 \times 3 \times 1$ supercell with both positive and negative atomic displacements $(\delta=0.03 \AA$ ). The diagonalization of the dynamical matrix provides phonon frequencies and phonon eigenvectors. For the $j$-th phonon mode, according to the Placzek approximation, the Raman intensity

$$
I \propto \frac{\left(n_{j}+1\right)}{\omega_{j}}\left|\boldsymbol{e}_{\mathbf{i}} \cdot \tilde{R} \cdot \boldsymbol{e}_{\mathbf{s}}^{\mathbf{T}}\right|^{2}
$$


where $\boldsymbol{e}_{\mathbf{i}}$ and $\boldsymbol{e}_{\mathrm{s}}$ are the electric polarization vectors of the incident and scattered lights respectively, and $\tilde{R}$ is the Raman tensor of the phonon mode. ${ }^{17} \omega_{j}$ is the frequency of the $j$-th phonon mode, and

$n_{j}=\left(e^{\hbar \omega_{j} / k_{B} T}-1\right)^{-1}$

is the Boltzmann distribution function. The matrix elements of the $(3 \times 3)$ Raman tensor $\tilde{R}$ are

$\tilde{R}_{\alpha \beta}(j)=\frac{V_{0}}{4 \pi} \sum_{\mu=1}^{N} \sum_{l=1}^{3} \frac{\partial \varepsilon_{\alpha \beta}}{\partial r_{l}(\mu)} \frac{e_{l}^{j}(\mu)}{\sqrt{M_{\mu}}}$

where $\varepsilon_{\alpha \beta}$ is the complex dielectric tensor depending on the excitation laser energy $E_{L}$, $r_{l}(\mu)$ is the position of the $\mu$-th atom along the direction $l, \frac{\partial \varepsilon_{\alpha \beta}}{\partial r_{l}(\mu)}$ is the derivative of the dielectric tensor over the atomic displacement, $\frac{e_{l}^{j}(\mu)}{\sqrt{M_{\mu}}}$ is the eigen-displacement of the $\mu$-th atom along the direction $l$ in the $j$-th phonon mode, $e_{l}^{j}(\mu)$ corresponds to the eigenvector of the dynamic matrix, $M_{\mu}$ is the mass of the $\mu$-th atom, and $V_{0}$ is the unit cell volume ${ }^{18}$ For both positive and negative atomic displacements $(\delta=0.03 \AA)$ in the unit cell, the complex dielectric tensors $\varepsilon_{\alpha \beta}$ were computed by DFT at the chosen laser energy and then the derivatives were obtained via the finite difference scheme. Based on the phonon eigenvectors and derivatives of dielectric tensors, the Raman tensor $\tilde{R}$ and subsequently Raman intensity of any phonon mode at any laser energy $E_{L}$ can be obtained.

Since DFT is well-known to underestimate the energy separations between the valence and conduction electronic states (including the band gap) $(13,14)$, the laser excitation energy of BP in DFT was multiplied by a scaling factor of 1.19. In this regard, DFT results often provide qualitative or even semi-quantitative agreement with experiments. More accurate calculations of REPs require inclusion of many-body electron-electron and electron-hole effects within the GW-BSE framework, which is too computationally challenging for the current case. 


\section{References}

(1) Mao, N.; Wu, J.; Han, B.; Lin, J.; Tong, L.; Zhang, J. Birefringence-Directed Raman Selection Rules in 2D Black Phosphorus Crystals. Small 2016, 12, 2627.

(2) Ling, X.; Huang, S.; Hasdeo, E. H.; Liang, L.; Parkin, W. M.; Tatsumi, Y.; Nugraha, A. R.; Puretzky, A. A.; Das, P. M.; Sumpter, B. G.; Geohegan, D. B.; Kong, J.; Saito, R.; Drndic, M.; Meunier, V.; Dresselhaus, M. S. Anisotropic Electron-Photon and Electron-Phonon Interactions in Black Phosphorus. Nano Lett. 2016, 16, 2260.

(3) Wu, J.; Mao, N.; Xie, L.; Xu, H.; Zhang, J. Identifying the Crystalline Orientation of Black Phosphorus Using Angle-Resolved Polarized Raman Spectroscopy. Angew. Chem. Int. Ed. 2015, 54, 2366.

(4) Kim, J.; Lee, J. U.; Lee, J.; Park, H. J.; Lee, Z.; Lee, C.; Cheong, H. Anomalous Polarization Dependence of Raman Scattering and Crystallographic Orientation of Black Phosphorus. Nanoscale 2015, 7, 18708.

(5) Ribeiro, H. B.; Pimenta, M. A.; de Matos, C. J.; Moreira, R. L.; Rodin, A. S.; Zapata, J. D.; de Souza, E. A.; Castro Neto, A. H. Unusual Angular Dependence of the Raman Response in Black Phosphorus. ACS Nano 2015, 9, 4270.

(6) Luo, Z.; Maassen, J.; Deng, Y.; Du, Y.; Garrelts, R. P.; Lundstrom, M. S.; Ye, P. D.; Xu, X. Anisotropic in-Plane Thermal Conductivity Observed in Few-Layer Black Phosphorus. Nat. Commun. 2015, 6, 8572.

(7) Wang, T.; Liu, J.; Xu, B.; Wang, R.; Yuan, P.; Han, M.; Xu, S.; Xie, Y.; Wu, Y.; Wang, X. Identifying the Crystalline Orientation of Black Phosphorus by Using Optothermal Raman Spectroscopy. ChemPhysChem 2017, 18, 2828.

(8) Ahmed, T.; Balendhran, S.; Karim, M. N.; Mayes, E. L. H.; Field, M. R.; Ramanathan, R.; Singh, M.; Bansal, V.; Sriram, S.; Bhaskaran, M.; Walia, S. Degradation of Black Phosphorus Is Contingent on UV-Blue Light Exposure. NPJ 2D. Mater. Appl. 2017, $1,18$.

(9) Guo, Z.; Zhang, H.; Lu, S.; Wang, Z.; Tang, S.; Shao, J.; Sun, Z.; Xie, H.; Wang, H.; Yu, X.-F.; Chu, P. K. From Black Phosphorus to Phosphorene: Basic Solvent Exfoliation, Evolution of Raman Scattering, and Applications to Ultrafast Photonics. Adv. Funct. Mater. 2015, 25, 6996.

(10) Yoon, D.; Moon, H.; Son, Y.-W.; Choi, J. S.; Park, B. H.; Cha, Y. H.; Kim, Y. D.; Cheong, H. Interference Effect on Raman Spectrum of Graphene on $\mathrm{SiO}_{2} / \mathrm{Si}$. Phys. Rev. B 2009, 80, 125422.

(11)Philipp, H. R. In Handbook of Optical Constants of Solids; Palik, E. D., Ed.; Academic Press: Burlington, 1997, p 749. 
(12)Asahina, H.; Morita, A. Band Structure and Optical Properties of Black Phosphorus. J. Phys. C: Solid State Phys. 1984, 17, 1839.

(13)Kresse, G.; Furthmüller, J. Efficiency of Ab-Initio Total Energy Calculations for Metals and Semiconductors Using a Plane-Wave Basis Set. Comput. Mater. Sci. 1996, 6, 15 .

(14)Perdew, J. P.; Burke, K.; Ernzerhof, M. Generalized Gradient Approximation Made Simple. Phys. Rev. Lett. 1996, 77, 3865.

(15)Dion, M.; Rydberg, H.; Schröder, E.; Langreth, D. C.; Lundqvist, B. I. Van Der Waals Density Functional for General Geometries. Phys. Rev. Lett. 2004, 92, 246401.

(16)Togo, A.; Oba, F.; Tanaka, I. First-Principles Calculations of the Ferroelastic Transition between Rutile-Type and $\mathrm{CaCl}_{2}$-Type $\mathrm{SiO}_{2}$ at High Pressures. Phys. Rev. B 2008, 78, 134106.

(17)Liang, L.; Meunier, V. First-Principles Raman Spectra of $\mathrm{MoS}_{2}, \mathrm{WS}_{2}$ and Their Heterostructures. Nanoscale 2014, 6, 5394.

(18)Talirz, L.; Söde, H.; Dumslaff, T.; Wang, S.; Sanchez-Valencia, J. R.; Liu, J.; Shinde, P.; Pignedoli, C. A.; Liang, L.; Meunier, V.; Plumb, N. C.; Shi, M.; Feng, X.; Narita, A.; Müllen, K.; Fasel, R.; Ruffieux, P. On-Surface Synthesis and Characterization of 9-Atom Wide Armchair Graphene Nanoribbons. ACS Nano 2017, 11, 1380. 